\title{
Photonic "hourglass" design for efficient quantum light emission
}

Østerkryger, Andreas Dyhl; Claudon, Julien; Gérard, Jean Michel; Gregersen, Niels

Published in:

Optics Letters

Link to article, DOI:

10.1364/OL.44.002617

Publication date:

2019

Document Version

Peer reviewed version

Link back to DTU Orbit

Citation (APA):

Østerkryger, A. D., Claudon, J., Gérard, J. M., \& Gregersen, N. (2019). Photonic "hourglass" design for efficient quantum light emission. Optics Letters, 44(11), 2617-2620. https://doi.org/10.1364/OL.44.002617

\section{General rights}

Copyright and moral rights for the publications made accessible in the public portal are retained by the authors and/or other copyright owners and it is a condition of accessing publications that users recognise and abide by the legal requirements associated with these rights.

- Users may download and print one copy of any publication from the public portal for the purpose of private study or research.

- You may not further distribute the material or use it for any profit-making activity or commercial gain

- You may freely distribute the URL identifying the publication in the public portal

If you believe that this document breaches copyright please contact us providing details, and we will remove access to the work immediately and investigate your claim. 


\title{
A photonic "hourglass" design for efficient quantum light emission
}

\author{
Andreas Dyhl Osterkryger ${ }^{1}$, Julien Claudon ${ }^{2}$, Jean-Michel Gérard ${ }^{2}$, and Niels \\ GREGERSEN ${ }^{1, *}$ \\ ${ }^{1}$ DTU Fotonik, Department of Photonics Engineering, Technical University of Denmark, DK-2800 Kongens Lyngby, Denmark \\ ${ }^{2}$ Univ. Grenoble Alpes, CEA, INAC, PHELIQS, "Nanophysique et semiconducteurs" group, F-38000 Grenoble, France \\ *Corresponding author: ngre@fotonik.dtu.dk
}

Compiled April 22, 2019

We propose a novel "hourglass"-shaped design for highly efficient generation and collection of quantum light. The design features a quantum dot in a photonic nanowire sandwiched between tapered Bragg reflectors. For a Purcell factor of 9, the design features a spontaneous emission coupling of 0.993 to the cavity mode enabled by the strong dielectric screening of radiation modes. Thanks to a highly reflecting bottom mirror, we furthermore demonstrate a collection efficiency of 0.95 to a Gaussian profile. Finally, this photonic structure features a broad operation bandwidth, as large as 11 nm. (c) 2019 Optical Society of America

OCIS codes: (140.3300) Laser beam shaping; (140.3948) Microcavity devices; (250.5590) Quantum-well, -wire and -dot devices.

\section{http://dx.doi.org/10.1364/ao.XX.XXXXXX}

Within optical quantum information technology [1], a key component is the quantum light source capable of emitting single photons and entangled photon pairs (EPPs) on demand. For scalable information processing, the source must feature both near-unity extraction efficiency and photon indistinguishability. For several decades, spontaneous parametric down-conversion [2] has been a workhorse for quantum light generation, however its probabilistic nature leads to efficiencies of only a few percent. On the other hand, the semiconductor quantum dot (QD) embedded in a structured environment [3] has in recent years emerged as a promising platform for realizing deterministic single-photon [4] and EPP sources [5]. Today, the most successful design strategy for single-photon generation is based on a QD in a microcavity pillar [6, 7], where Purcell enhancement is exploited to ensure high efficiency. Here, a photon collection efficiency $\varepsilon$ up to 0.79 [8] has been demonstrated, and more recently an $\varepsilon$ of 0.66 combined with 0.985 photon indistinguishability [9] was reported. However, the micropillar design approach is inherently narrowband (bandwidth $\Delta \lambda \sim 0.2 \mathrm{~nm}$ ), whereas EPP generation using the exciton-biexciton cascade requires a bandwidth of typically a few $\mathrm{nm}$. While EPP generation from a double-pillar "molecule" geometry [10] with an extraction efficiency $\varepsilon$ of 0.35 has been demonstrated, this design suffers from a highly non-Gaussian far-field emission pattern. An alterna- tive design approach consists in placing the QD in a photonic nanowire, where a dielectric screening effect [11] is employed to ensure high efficiency. In particular, no resonant effect is used and the design approach is thus broadband. Single-photon generation efficiency $\varepsilon$ up to 0.75 from "needle" [12] and "trumpet" nanowires [13-15] has been demonstrated as well as EPP generation $[16,17]$ with $\varepsilon$ of 0.15 . However, obtaining high photon indistinguishability from photonic nanowires remains a challenge. Known decoherence mechanisms include fast exciton energy fluctuations due to an unstable electrostatic environment [18] and well as interaction with phonons [19] and discrete vibration modes of the nanowire [20]. While resonant effects are not needed for high efficiency, it is nevertheless desirable to enhance the spontaneous emission (SE) rate to overcome [19] these decoherence mechanisms and ensure high indistinguishability. To this purpose it was recently suggested to introduce weak cavity effects by implementing a distributed Bragg reflector (DBR) in the "trumpet" taper [21]. Here the cavity consists of a bottom silica-gold mirror [22] and the top DBR. A bottleneck of this design is the bottom metal mirror with reflectivity limited to $\sim 0.91$ [21-23]. In particular, when the SE is Purcell enhanced in the presence of the DBR, this occurs at the cost of reduced efficiency due to increased light absorption in the metal.

To avoid this trade-off and to pave the way towards combined near-unity efficiency and indistinguishability, we propose in this Letter the "hourglass" design illustrated in Fig. 1(a). Its novelty consists of the combination of a top "trumpet" taper featuring a DBR [21] and a bottom inverted "trumpet" taper featuring another DBR [24] allowing for near-unity bottom mirror reflectivity. We will show that the "hourglass" may overcome the trade-offs of present design strategies both in terms of efficiency and indistinguishability [25]. In the following, we first discuss the single-mode model used to analyze the performance of the "hourglass". Subsequently, we discuss the achievable reflectivity of the bottom DBR mirror. After having fixed a geometry, we then present calculations of the Purcell factor $F_{\mathrm{P}}$, the cavity coupling factor $\beta_{\mathrm{c}}$, the collection efficiency $\varepsilon$ and the bandwidth $\Delta \lambda$. We will demonstrate that the "hourglass" allows for an $\varepsilon$ of 0.95 combined with a $\Delta \lambda$ of $11 \mathrm{~nm}$ and an $F_{\mathrm{P}}$ of 9 . Finally, we discuss strategies for further increasing the efficiency towards unity.

In this Letter, we consider a rotationally symmetric structure suitable for the EPP application. The radius at the position of the 


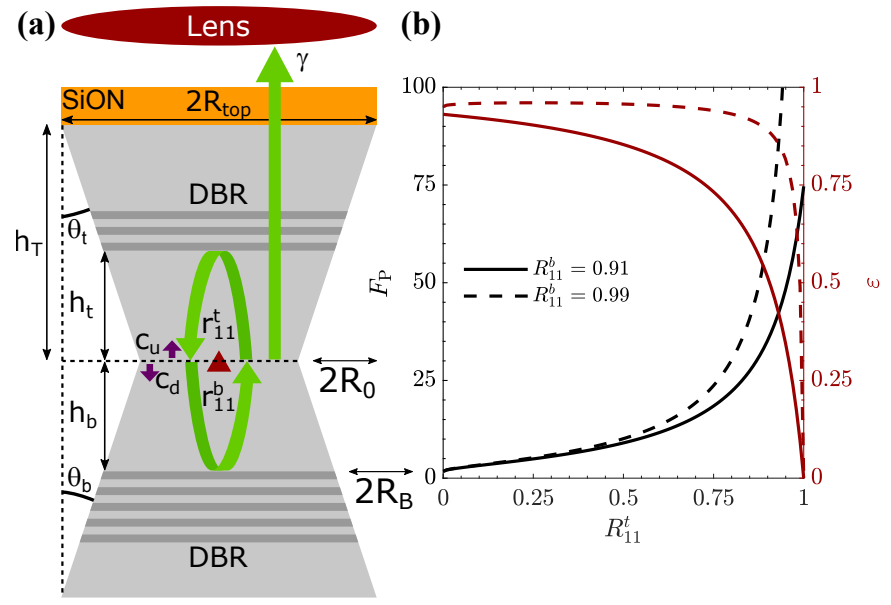

Fig. 1. (a) The "hourglass" design. (b) Purcell enhancement and total efficiency calculated using a single-mode model as function of the top mirror reflectivity for $R_{11}^{b}=0.91$ (solid) and $R_{11}^{\mathrm{b}}=0.99$ (dashed). The parameters used are $\gamma=0.975\left(1-R_{11}^{t}\right), \Gamma_{\mathrm{HE}_{11}}=0.88 \Gamma_{0}$ and $\Gamma_{\mathrm{Rad}}=0.04 \Gamma_{0}[21]$.

QD is chosen as $R_{0}=0.114 \mu \mathrm{m}$ to ensure good coupling from the QD to the fundamental waveguide mode for our design wavelength $\lambda_{\mathrm{d}}=925 \mathrm{~nm}$ as discussed below. The cavity is formed by double "trumpets" with GaAs/AlGaAs DBRs featuring linear tapering profiles with side wall angles $\theta_{\mathrm{b}}$ and $\theta_{\mathrm{t}}$ and QD-DBR separation distances $h_{\mathrm{b}}$ and $h_{\mathrm{t}}$. The thickness of the DBR layers is optimized using the procedure outlined in Appendix C of Ref. [21]. In addition, the design features a silicon oxynitride ( $\mathrm{SiON}$ ) [26] anti-reflecting (AR) coating. The refractive indices are those of Ref. [21] except for the AR coating for which we have chosen $\sqrt{n_{\mathrm{GaAs}}}$. In the following, we investigate a symmetric structure such that $\theta_{\mathrm{b}}=\theta_{\mathrm{t}} \equiv \theta$ and $h_{\mathrm{b}}=h_{\mathrm{t}} \equiv h$. The numerical simulations are performed using a Fourier modal method [27] with a true open geometry boundary condition combined with a standard scattering matrix formalism [28].

We present results both from exact calculations using a full model as well as from the single-mode model of Ref. [21], which is restated here for clarity. We consider the propagation of the fundamental guided $\mathrm{HE}_{11}$ mode in the different sections of the "hourglass" structure as sketched in Fig. 1(a). We start by considering an infinitely long uniform wire. Here, the fraction of QD SE funnelled into the guided mode is $\beta_{\mathrm{g}} \equiv \Gamma_{\mathrm{HE}_{11}} /\left(\Gamma_{\mathrm{HE}_{11}}+\Gamma_{\mathrm{Rad}}\right)$, where $\Gamma_{\mathrm{HE}_{11}}\left(\Gamma_{\mathrm{Rad}}\right)$ is the SE rate into the $\mathrm{HE}_{11}$ mode (radiation modes) of the uniform nanowire. $\beta_{\mathrm{g}}$ reaches a maximum of 0.96 [21] for a wire radius $R_{0}=114 \mathrm{~nm}$. We now consider the full "hourglass" geometry, where we define $c_{u(d)}$ as the complex amplitude coefficient of the fundamental upward (downward) propagating mode given by

$$
c_{\mathrm{u}}=c_{0} \frac{1+r_{11}^{\mathrm{b}}}{1-r_{11}^{\mathrm{b}} r_{11}^{\mathrm{t}}}, \quad c_{\mathrm{d}}=c_{0} \frac{1+r_{11}^{\mathrm{t}}}{1-r_{11}^{\mathrm{b}} r_{11}^{\mathrm{t}}},
$$

where $c_{0}=\sqrt{\Gamma_{\mathrm{HE}_{11}} / 2}$ is the $\mathrm{HE}_{11}$ amplitude coefficient for an infinitely long nanowire, and $r_{11}^{\mathrm{b}}$ and $r_{11}^{\mathrm{t}}$ are complex modal reflection coefficients of the bottom and top mirrors, respectively, including propagation phases between the QD and the first $\mathrm{AlGaAs}$ layer. The total SE rate $\Gamma_{\mathrm{T}}$ of the full geometry is then computed as

$$
\Gamma_{\mathrm{T}}=\left(1-\left|r_{11}^{\mathrm{t}}\right|^{2}\right)\left|c_{\mathrm{u}}\right|^{2}+\left(1-\left|r_{11}^{\mathrm{b}}\right|^{2}\right)\left|c_{\mathrm{d}}\right|^{2}+\Gamma_{\mathrm{Rad}}
$$

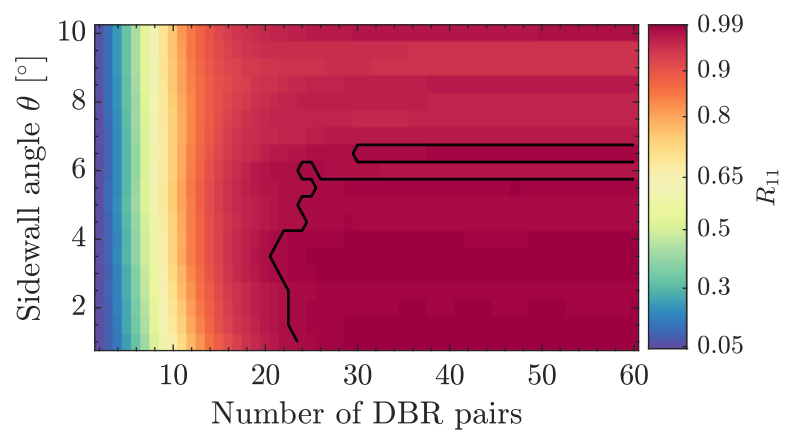

Fig. 2. Bottom mirror reflectivity $R_{11}^{b}$ as function of number of DBR pairs and sidewall angle $\theta$. The first DBR layer is placed at a distance $h_{\mathrm{b}}$ below the QD, such that the radius of the first DBR layer is $R_{\mathrm{B}}=150 \mathrm{~nm}$. The black contour line indicates 0.98 reflectivity.

and furthermore we define a generalized Purcell factor as

$$
F_{\mathrm{P}}=\operatorname{Re}\left[\frac{\left(1+r_{11}^{\mathrm{t}}\right)\left(1+r_{11}^{\mathrm{b}}\right)}{1-r_{11}^{\mathrm{t}} r_{11}^{\mathrm{b}}}\right] \frac{\Gamma_{\mathrm{HE}_{11}}}{\Gamma_{0}},
$$

where $\Gamma_{0}$ is the bulk SE rate. This generalized Purcell factor should be understood as the local density of states at the position of the emitter and correctly describes the normalized SE rate into the cavity mode not only at resonance but at all wavelengths as discussed in detail in Ref. [21]. The cavity SE coupling factor is defined using the Purcell factor as $\beta_{\mathrm{c}}=F_{\mathrm{P}} \Gamma_{0} / \Gamma_{\mathrm{T}}$. Finally, we define the efficiency $\varepsilon$ as the ratio of the power coupled to a Gaussian mode in the far field $[13,14]$ collected using a lens with 0.8 numerical aperture. It is given by

$$
\varepsilon=\gamma\left|c_{\mathrm{u}}\right|^{2} / \Gamma_{\mathrm{T}}
$$

where $\gamma$ is the total transmission of the fundamental mode from the QD to the first lens including the overlap with a Gaussian profile $[13,14]$.

To understand the impact of a highly reflecting bottom mirror we present the collection efficiency (Eq. (4)) and the Purcell enhancement (Eq. (3)) as function of the top mirror reflectivity $R_{11}^{t}=\left|r_{11}^{t}\right|^{2}$ in Fig. 1(b) for bottom mirror reflectivities $R_{11}^{b}=$ $\left|r_{11}^{b}\right|^{2}$ of 0.91 and 0.99 . For a reflectivity of 0.91 corresponding to the silica-gold mirror, we observe a clear compromise between obtaining simultaneously high Purcell enhancement and high collection efficiency. However, for $R_{11}^{b}=0.99$ we can obtain a Purcell factor above 20 without sacrificing collection efficiency. Clearly, the implementation of a bottom mirror with near-unity reflectivity is crucial in the pursuit of quantum light sources for scalable quantum information processing.

We now present the calculated modal reflectivity $R_{11}^{b}$ of the tapered DBR bottom mirror as function of the sidewall angle $\theta$ and the number of DBR layer pairs in Fig. 2. Since the reflectivity of the DBR is significantly compromised for nanowire radii below $\sim 0.15 \lambda$ [22], we have chosen to place the first DBR pair at a QD-DBR height $h$ corresponding to a radius $R_{\mathrm{B}}$ of $150 \mathrm{~nm}$. We observe that a reflectivity above 0.98 is achievable for a sidewall angle $\theta$ below $6^{\circ}$, and the general trend is that the reflectivity improves with decreasing angle and increasing number of layer pairs. Additionally, careful inspection reveals oscillations in the reflectivity as function of the sidewall angle. These oscillations are due to coupling between the fundamental mode and higher 


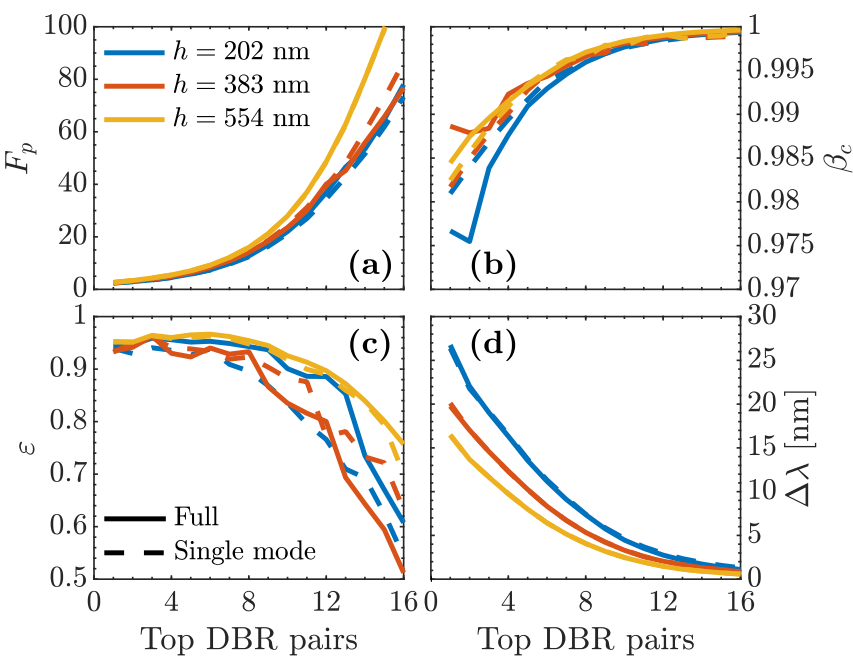

Fig. 3. Purcell enhancement $F_{\mathrm{P}}(\mathrm{a})$, cavity SE coupling factor $\beta_{\mathrm{c}}(\mathrm{b})$, collection efficiency $\varepsilon$ (c) and bandwidth $\Delta \lambda$ of the Purcell enhancement (d) as function of the number of DBR pairs in the top mirror computed for the first three resonant QDDBR separation distances using the full model (solid curves) and the single-mode model (dashed curves).

order modes at the position of the first AlGaAs layer and could be suppressed by increasing $R_{\mathrm{B}}$ or by introducing an adiabatic DBR profile [29] with a gradually increasing AlGaAs layer thickness and/or a gradually decreasing $\mathrm{AlGaAs}$ refractive index.

A small sidewall angle $\theta$ is required for adiabatic propagation [23] of the $\mathrm{HE}_{11}$ mode through the taper, and a large top radius $R_{\text {Top }}$ is needed to ensure a low output beam divergence [13-15] and a good coupling to a Gaussian mode in the far field. However, a large $R_{\text {Top }}$ and a small $\theta$ lead to a large top taper height $h_{\mathrm{T}}$, whereas a compact device is preferential for the fabrication. There is thus a trade-off between high performance and choosing an $h_{\mathrm{T}}$ compatible with practical fabrication constraints. In the following, we chose $h_{\mathrm{T}}=15 \mu \mathrm{m}$ and $R_{\mathrm{Top}}=1.2 \mu \mathrm{m}$ corresponding to a $\theta$ of $4.1^{\circ}$, which are parameters well compatible [15] with feasible fabrication. With this choice of parameters we compute a transmission $\gamma$ of 0.975 to a Gaussian profile for the bare taper geometry, i.e. with no DBR in the top taper. Furthermore, we chose 40 layer pairs in the bottom DBR, which for $R_{\mathrm{B}}=$ $150 \mathrm{~nm}$ leads to a bottom reflectivity $R_{11}^{b}$ of 0.987 - a remarkable improvement in reflectivity as compared to the dielectric-metal mirror. In the following, we investigate the influence of the number of layer pairs in the top DBR and of the QD-DBR separation $h$. The maximum Purcell enhancement occurs when the QD is positioned at an antinode of the cavity mode, which is the case for the discrete values of $h$ for which the total phase $\arg \left(r_{11}^{\mathrm{t}} r_{11}^{\mathrm{b}}\right)$ describing a cavity roundtrip is zero. We note that increasing the number of DBR layer pairs from 4 to 40 modifies the total phase by less than $0.5^{\circ}$, which justifies our choice of identical top and bottom QD-DBR separation distances.

We present the computed Purcell enhancement $F_{\mathrm{P}}$, cavity coupling factor $\beta_{\mathrm{c}}$, collection efficiency $\varepsilon$ and bandwidth $\Delta \lambda$ of the Purcell enhancement in Fig. 3 as function of the number of top DBR layer pairs. The bandwidth is determined by computing the $Q$ factor using the approach given in Ref. [30]. We present results for the first three separations $h$ for which the emitter is located at an antinode at the design wavelength of $\lambda_{\mathrm{d}}=925 \mathrm{~nm}$. We observe that the Purcell factor increases slightly with larger

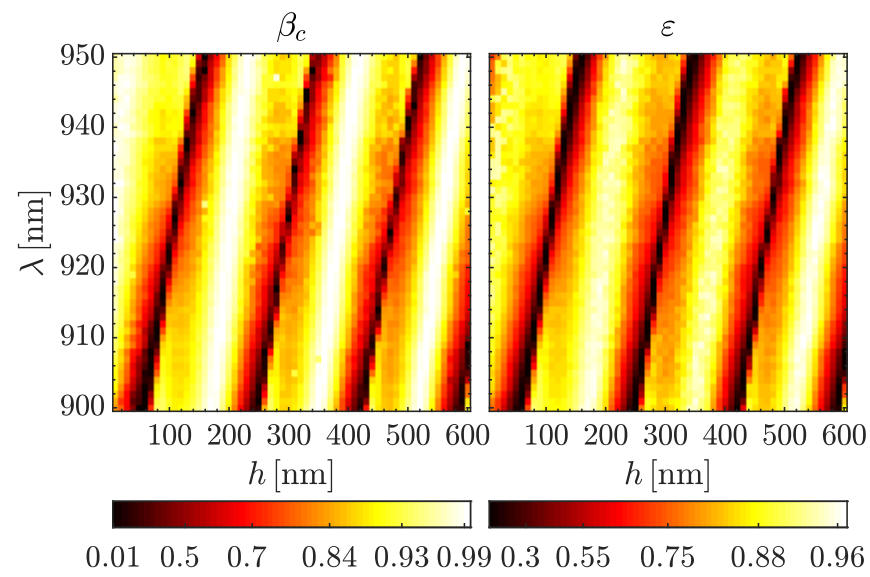

Fig. 4. Cavity coupling factor $\beta_{\mathrm{c}}$ and collection efficiency $\varepsilon$ as function of emission wavelength $\lambda$ and QD-DBR separation distance $h$ with $\theta=4.1^{\circ}, R_{\text {Top }}=1.2 \mu \mathrm{m}, h_{\mathrm{T}}=15 \mu \mathrm{m}$ and 40 (6) layer pairs in the bottom (top) DBR.

separation $h$. As the trumpet radius increases, the $\mathrm{HE}_{11}$ modal overlap at the GaAs/AlGaAs interfaces is improved, and this in turn leads to increased reflectivity [21] and Purcell enhancement. The cavity coupling factor $\beta_{\mathrm{c}}$ exceeds 0.97 even for a weakly reflecting DBR, which is a result of dielectric screening by the nanowire [11]. The collection efficiency $\varepsilon$ is slightly improved with $h$ due to the increase in Purcell enhancement, and it remains above $\sim 0.9$ for up to 8 layer pairs thanks to the high reflectivity of the bottom DBR. As expected the bandwidth is largest for the shortest cavity, for which we observe that a $\Delta \lambda$ of $11 \mathrm{~nm}$ is obtained for 6 layer pairs. Fig. 3 includes results obtained from the single-mode model described above as well as from a full model taking into account interaction of the $\mathrm{HE}_{11}$ mode with radiation modes in the evaluation of the amplitude coefficients $c_{u}$ and $c_{d}$. Whereas the agreement for $F_{\mathrm{P}}$ and $\Delta \lambda$ for two models is good, discrepancies are observed for $\beta_{\mathrm{c}}$ and $\varepsilon$ which increase with reduced QD-DBR separation $h$. This discrepancy originates from a modified $c_{u}$ coefficient for the smaller cavities due to interaction with radiation modes. Whereas the single-mode model has provided remarkable agreement for simpler designs $[23,24]$, we conclude that full simulations are needed to predict the performance in the presence of significant cavity effects.

We now investigate the performance of an "hourglass" design featuring 40 (6) pairs in the bottom (top) DBR. The Purcell factor for this design is slightly larger than for the case with the silicagold mirror [21] due to the increased reflectivity of the bottom DBR with a maximum $F_{\mathrm{P}}$ of $\sim 9$ at the design wavelength and a corresponding $\beta_{\mathrm{c}}$ of 0.993 . Furthermore, efficiencies $\varepsilon$ of 0.95 and 0.965 are obtained for $h=202$ and $554 \mathrm{~nm}$ respectively - significant improvements compared to the $\varepsilon$ of 0.8 reported in Ref. [21]. While keeping the design wavelength $\lambda_{d}=925 \mathrm{~nm}$ fixed, we then present the cavity coupling factor $\beta_{\mathrm{c}}$ and the collection efficiency $\varepsilon$ in Fig. 4 as function of emitter wavelength $\lambda$ and QD-DBR separation distance $h$. We observe that a $\beta_{\mathrm{c}}$ above 0.95 is obtained over a $36 \mathrm{~nm}$ range for the first resonance line highlighting the broadband nature of the design in terms of efficient coupling to the cavity mode. This leads to a collection efficiency above 0.88 over the same range for $h=202 \mathrm{~nm}$. Similarly, Fig. 4 demonstrates the good tolerance of $\beta_{\mathrm{c}}$ and $\varepsilon$ with respect to $h$ thus ensuring feasibility in the fabrication. Finally, we note that, for $h=202 \mathrm{~nm}$, the 0.95 collection efficiency to a Gaussian mode 
is combined with an $11 \mathrm{~nm}$ bandwidth, which makes the design attractive for the EPP application. The combination of the high Purcell factor with the broad spectral bandwidth is achieved thanks to a small cavity mode volume of $\sim 0.7(\lambda / n)^{3}[21]$.

Thus the "hourglass" design clearly has potential to outperform the micropillar geometry in the EPP application, and we now consider its strengths within the single-photon source application, where the micropillar geometry currently represents state-of-the-art. Here, the rotationally symmetric micropillar design suffers from lack of polarization control, which reduces its efficiency by a factor of 2 due to polarization filtering. An elliptical cross-section has been suggested [7] to establish polarization control, however it necessarily reduces the overlap to a Gaussian profile in the far field leading to a trade-off between degree of polarization and efficiency. On the other hand, the "hourglass" allows for an elliptical cross-section [31] at the position of the emitter combined with a rotationally symmetric top termination thus enabling for polarization control without compromising the far field pattern. Additionally, we note that the micropillar relies on Purcell enhancement to ensure high efficiency, and it is thus ultimately limited by a trade-off [25] between efficiency and indistinguishability in the presence of phonon-induced decoherence. Whereas calculations of the indistinguishability are beyond the scope of this Letter, we remark that the above tradeoff is avoided in the "hourglass" design as high efficiency is obtained independently of the Purcell factor, which instead may be chosen to maximize the indistinguishability.

Whereas an exhaustive parameter optimization for the "hourglass" design is beyond the scope of this Letter, we finally now qualitatively point out strategies for increasing the efficiency further towards unity. Three main factors limit the collection efficiency of the present design: 1) A DBR operating in a lowdiameter regime suffers from scattering [32] to higher order modes. As discussed above, this scattering can be suppressed using adiabatic engineering [29] of the layer thicknesses and of the refractive index profile. 2) The side wall angle $\theta$ of $4.1^{\circ}$ can be reduced further to increase $\gamma$ for the bare taper. The price to pay is an increased top taper height $h_{\mathrm{T}}$. We have chosen a modest $h_{\mathrm{T}}$ of $15 \mu \mathrm{m}$, however top tapers of almost twice this height [15] have already been demonstrated experimentally. 3) The maximum overlap between a $\mathrm{HE}_{11}$ mode in a GaAs nanowire (Bessel profile) and an optical fibre (Gaussian profile) is 0.985 . This limitation, which also applies to the micropillar, can be overcome by butt-coupling the source to a tapered optical fiber.

In conclusion, we have proposed an "hourglass"-shaped design for a highly efficient source of quantum light. For a modest taper height compatible with fabrication, we show that an efficiency of 0.95 is achievable combined with an $11 \mathrm{~nm}$ bandwidth. The design does not rely on Purcell enhancement to achieve high efficiency, and the Purcell factor can be freely chosen e.g. to maximize the photon indistinguishability.

Funding. QuantERA ERA-NET Cofund (HYPER-U-P-S); Innovation Fund Denmark (7074-00004B); Independent Research Fund Denmark (DFF-4181-00416); French National Research Agency (ANR-16-CE09-0010-01); CEA DRF Impulsion (SOUPAPE).

\section{REFERENCES}

1. S. Barz, J. Phys. B At. Mol. Opt. Phys. 48, 083001 (2015).

2. P. G. Kwiat, K. Mattle, H. Weinfurter, A. Zeilinger, A. V. Sergienko, and Y. Shih, Phys. Rev. Lett. 75, 4337 (1995).

3. P. Lodahl, S. Mahmoodian, and S. Stobbe, Rev. Mod. Phys. 87, 347 (2015).
4. I. Aharonovich, D. Englund, and M. Toth, Nat. Photonics 10, 631 (2016).

5. D. Huber, M. Reindl, J. Aberl, A. Rastelli, and R. Trotta, J. Opt. 20, 073002 (2018).

6. S. Reitzenstein and A. Forchel, J. Phys. D. Appl. Phys. 43, 033001 (2010).

7. E. Moreau, I. Robert, J. M. Gérard, I. Abram, L. Manin, and V. ThierryMieg, Appl. Phys. Lett. 79, 2865 (2001).

8. O. Gazzano, S. Michaelis de Vasconcellos, C. Arnold, A. Nowak, E. Galopin, I. Sagnes, L. Lanco, A. Lemaître, and P. Senellart, Nat. Commun. 4, 1425 (2013).

9. X. Ding, Y. He, Z.-C. Duan, N. Gregersen, M.-C. Chen, S. Unsleber, S. Maier, C. Schneider, M. Kamp, S. Höfling, C.-Y. Lu, and J.-W. Pan, Phys. Rev. Lett. 116, 020401 (2016).

10. A. Dousse, J. Suffczyński, A. Beveratos, O. Krebs, A. Lemaître, I. Sagnes, J. Bloch, P. Voisin, and P. Senellart, Nature. 466, 217 (2010).

11. J. Bleuse, J. Claudon, M. Creasey, N. S. Malik, J.-M. Gérard, I. Maksymov, J.-P. Hugonin, and P. Lalanne, Phys. Rev. Lett. 106, 103601 (2011).

12. J. Claudon, J. Bleuse, N. S. Malik, M. Bazin, P. Jaffrennou, N. Gregersen, C. Sauvan, P. Lalanne, and J.-M. Gérard, Nat. Photonics 4, 174 (2010).

13. M. Munsch, N. S. Malik, E. Dupuy, A. Delga, J. Bleuse, J.-M. Gérard, J. Claudon, N. Gregersen, and J. Mørk, Phys. Rev. Lett. 110, 177402 (2013).

14. M. Munsch, N. S. Malik, E. Dupuy, A. Delga, J. Bleuse, J.-M. Gérard, J. Claudon, N. Gregersen, and J. Mørk, Phys. Rev. Lett. 111, 239902 (2013).

15. P. Stepanov, A. Delga, N. Gregersen, E. Peinke, M. Munsch, J. Teissier, J. Mørk, M. Richard, J. Bleuse, J.-M. Gérard, and J. Claudon, Appl. Phys. Lett. 107, 141106 (2015).

16. T. Huber, A. Predojević, M. Khoshnegar, D. Dalacu, P. J. Poole, H. Majedi, and G. Weihs, Nano Lett. 14, 7107 (2014).

17. M. A. M. Versteegh, M. E. Reimer, K. D. Jöns, D. Dalacu, P. J. Poole, A. Gulinatti, A. Giudice, and V. Zwiller, Nat. Commun. 5, 5298 (2014).

18. A. Berthelot, I. Favero, G. Cassabois, C. Voisin, C. Delalande, P. Roussignol, R. Ferreira, and J.-M. Gérard, Nat. Phys. 2, 759 (2006).

19. P. Kaer, N. Gregersen, and J. Mørk, New J. Phys. 15, 035027 (2013).

20. M. Munsch, A. V. Kuhlmann, D. Cadeddu, J.-M. Gérard, J. Claudon, M. Poggio, and R. J. Warburton, Nat. Commun. 8, 76 (2017).

21. N. Gregersen, D. P. S. McCutcheon, J. Mørk, J.-M. Gérard, and J. Claudon, Opt. Express 24, 20904 (2016).

22. I. Friedler, P. Lalanne, J. P. Hugonin, J. Claudon, J. M. Gérard, A. Beveratos, and I. Robert-Philip, Opt. Lett. 33, 2635 (2008).

23. N. Gregersen, T. R. Nielsen, J. Mørk, J. Claudon, and J.-M. Gérard, Opt. Express 18, 21204 (2010).

24. I. Friedler, C. Sauvan, J. P. Hugonin, P. Lalanne, J. Claudon, and J. M. Gérard, Opt. Express 17, 2095 (2009).

25. J. Iles-Smith, D. P. S. McCutcheon, A. Nazir, and J. Mørk, Nat. Photonics 11, 521 (2017).

26. R. Machorro, E. Samano, G. Soto, F. Villa, and L. Cota-Araiza, Mater. Lett. 45, 47 (2000).

27. T. Häyrynen, J. R. de Lasson, and N. Gregersen, J. Opt. Soc. Am. A 33, 1298 (2016).

28. A. V. Lavrinenko, J. Lægsgaard, N. Gregersen, F. Schmidt, and T. Søndergaard, "Numerical Methods in Photonics," (CRC Press, 2015), chap. 6, pp. 139-195.

29. M. Lermer, N. Gregersen, F. Dunzer, S. Reitzenstein, S. Höfling, J. Mørk, L. Worschech, M. Kamp, and A. Forchel, Phys. Rev. Lett. 108, 057402 (2012).

30. N. Gregersen, S. Reitzenstein, C. Kistner, M. Strauss, C. Schneider, S. Höfling, L. Worschech, A. Forchel, T. R. Nielsen, J. Mørk, and J.-M. Gérard, IEEE J. Quantum Electron. 46, 1470 (2010).

31. M. Munsch, J. Claudon, J. Bleuse, N. S. Malik, E. Dupuy, J.-M. Gérard, Y. Chen, N. Gregersen, and J. Mørk, Phys. Rev. Lett. 108, 077405 (2012).

32. P. Lalanne, J. P. Hugonin, and J. M. Gérard, Appl. Phys. Lett. 84, 4726 (2004). 


\section{FULL REFERENCES}

1. S. Barz, "Quantum computing with photons: introduction to the circuit model, the one-way quantum computer, and the fundamental principles of photonic experiments," J. Phys. B At. Mol. Opt. Phys. 48, 083001 (2015).

2. P. G. Kwiat, K. Mattle, H. Weinfurter, A. Zeilinger, A. V. Sergienko, and Y. Shih, "New High-Intensity Source of Polarization-Entangled Photon Pairs," Phys. Rev. Lett. 75, 4337-4341 (1995).

3. P. Lodahl, S. Mahmoodian, and S. Stobbe, "Interfacing single photons and single quantum dots with photonic nanostructures," Rev. Mod. Phys. 87, 347-400 (2015).

4. I. Aharonovich, D. Englund, and M. Toth, "Solid-state single-photon emitters," Nat. Photonics 10, 631-641 (2016).

5. D. Huber, M. Reindl, J. Aberl, A. Rastelli, and R. Trotta, "Semiconductor quantum dots as an ideal source of polarization-entangled photon pairs on-demand: a review," J. Opt. 20, 073002 (2018).

6. S. Reitzenstein and A. Forchel, "Quantum dot micropillars," J. Phys. D. Appl. Phys. 43, 033001 (2010).

7. E. Moreau, I. Robert, J. M. Gérard, I. Abram, L. Manin, and V. ThierryMieg, "Single-mode solid-state single photon source based on isolated quantum dots in pillar microcavities," Appl. Phys. Lett. 79, 2865-2867 (2001).

8. O. Gazzano, S. Michaelis de Vasconcellos, C. Arnold, A. Nowak, E. Galopin, I. Sagnes, L. Lanco, A. Lemaître, and P. Senellart, "Bright solidstate sources of indistinguishable single photons," Nat. Commun. 4, 1425 (2013).

9. X. Ding, Y. He, Z.-C. Duan, N. Gregersen, M.-C. Chen, S. Unsleber, S. Maier, C. Schneider, M. Kamp, S. Höfling, C.-Y. Lu, and J.-W. Pan, "On-Demand Single Photons with High Extraction Efficiency and NearUnity Indistinguishability from a Resonantly Driven Quantum Dot in a Micropillar," Phys. Rev. Lett. 116, 020401 (2016).

10. A. Dousse, J. Suffczyński, A. Beveratos, O. Krebs, A. Lemaître, I. Sagnes, J. Bloch, P. Voisin, and P. Senellart, "Ultrabright source of entangled photon pairs," Nature. 466, 217-220 (2010).

11. J. Bleuse, J. Claudon, M. Creasey, N. S. Malik, J.-M. Gérard, I. Maksymov, J.-P. Hugonin, and P. Lalanne, "Inhibition, enhancement, and control of spontaneous emission in photonic nanowires," Phys. Rev. Lett. 106, 103601 (2011).

12. J. Claudon, J. Bleuse, N. S. Malik, M. Bazin, P. Jaffrennou, N. Gregersen, C. Sauvan, P. Lalanne, and J.-M. Gérard, "A highly efficient single-photon source based on a quantum dot in a photonic nanowire," Nat. Photonics 4, 174-177 (2010).

13. M. Munsch, N. S. Malik, E. Dupuy, A. Delga, J. Bleuse, J.-M. Gérard, J. Claudon, N. Gregersen, and J. Mørk, "Dielectric GaAs antenna ensuring an efficient broadband coupling between an InAs quantum dot and a Gaussian optical beam," Phys. Rev. Lett. 110, 177402 (2013).

14. M. Munsch, N. S. Malik, E. Dupuy, A. Delga, J. Bleuse, J.-M. Gérard, J. Claudon, N. Gregersen, and J. Mørk, "Erratum: Dielectric GaAs Antenna Ensuring an Efficient Broadband Coupling between an InAs Quantum Dot and a Gaussian Optical Beam [Phys. Rev. Lett. 110, 177402 (2013)]," Phys. Rev. Lett. 111, 239902 (2013).

15. P. Stepanov, A. Delga, N. Gregersen, E. Peinke, M. Munsch, J. Teissier, J. Mørk, M. Richard, J. Bleuse, J.-M. Gérard, and J. Claudon, "Highly directive and Gaussian far-field emission from "giant" photonic trumpets," Appl. Phys. Lett. 107, 141106 (2015).

16. T. Huber, A. Predojević, M. Khoshnegar, D. Dalacu, P. J. Poole, H. Majedi, and G. Weihs, "Polarization Entangled Photons from Quantum Dots Embedded in Nanowires," Nano Lett. 14, 7107-7114 (2014).

17. M. A. M. Versteegh, M. E. Reimer, K. D. Jöns, D. Dalacu, P. J. Poole, A. Gulinatti, A. Giudice, and V. Zwiller, "Observation of strongly entangled photon pairs from a nanowire quantum dot," Nat. Commun. 5, 5298 (2014).

18. A. Berthelot, I. Favero, G. Cassabois, C. Voisin, C. Delalande, P. Roussignol, R. Ferreira, and J.-M. Gérard, "Unconventional motional narrowing in the optical spectrum of a semiconductor quantum dot," Nat. Phys. 2, 759-764 (2006).

19. P. Kaer, N. Gregersen, and J. Mørk, "The role of phonon scattering in the indistinguishability of photons emitted from semiconductor cavity
QED systems," New J. Phys. 15, 035027 (2013).

20. M. Munsch, A. V. Kuhlmann, D. Cadeddu, J.-M. Gérard, J. Claudon, M. Poggio, and R. J. Warburton, "Resonant driving of a single photon emitter embedded in a mechanical oscillator," Nat. Commun. 8, 76 (2017).

21. N. Gregersen, D. P. S. McCutcheon, J. Mørk, J.-M. Gérard, and J. Claudon, "A broadband tapered nanocavity for efficient nonclassical light emission," Opt. Express 24, 20904-20924 (2016).

22. I. Friedler, P. Lalanne, J. P. Hugonin, J. Claudon, J. M. Gérard, A. Beveratos, and I. Robert-Philip, "Efficient photonic mirrors for semiconductor nanowires," Opt. Lett. 33, 2635-2637 (2008).

23. N. Gregersen, T. R. Nielsen, J. Mørk, J. Claudon, and J.-M. Gérard, "Designs for high-efficiency electrically pumped photonic nanowire single-photon sources," Opt. Express 18, 21204-21218 (2010).

24. I. Friedler, C. Sauvan, J. P. Hugonin, P. Lalanne, J. Claudon, and J. M. Gérard, "Solid-state single photon sources: the nanowire antenna," Opt. Express 17, 2095-2110 (2009).

25. J. Iles-Smith, D. P. S. McCutcheon, A. Nazir, and J. Mørk, "Phonon scattering inhibits simultaneous near-unity efficiency and indistinguishability in semiconductor single-photon sources," Nat. Photonics 11, 521-526 (2017).

26. R. Machorro, E. Samano, G. Soto, F. Villa, and L. Cota-Araiza, "Modification of refractive index in silicon oxynitride films during deposition," Mater. Lett. 45, 47-50 (2000)

27. T. Häyrynen, J. R. de Lasson, and N. Gregersen, "Open-geometry Fourier modal method: Modeling nanophotonic structures in infinite domains," J. Opt. Soc. Am. A 33, 1298-1306 (2016).

28. A. V. Lavrinenko, J. Lægsgaard, N. Gregersen, F. Schmidt, and T. Søndergaard, "Numerical Methods in Photonics," (CRC Press, 2015), chap. 6, pp. 139-195.

29. M. Lermer, N. Gregersen, F. Dunzer, S. Reitzenstein, S. Höfling, J. Mørk, L. Worschech, M. Kamp, and A. Forchel, "Bloch-wave engineering of quantum dot micropillars for cavity quantum electrodynamics experiments," Phys. Rev. Lett. 108, 057402 (2012).

30. N. Gregersen, S. Reitzenstein, C. Kistner, M. Strauss, C. Schneider, S. Höfling, L. Worschech, A. Forchel, T. R. Nielsen, J. Mørk, and J.-M. Gérard, "Numerical and experimental study of the $Q$ factor of high-Q micropillar cavities," IEEE J. Quantum Electron. 46, 1470-1483 (2010).

31. M. Munsch, J. Claudon, J. Bleuse, N. S. Malik, E. Dupuy, J.-M. Gérard, Y. Chen, N. Gregersen, and J. Mørk, "Linearly polarized, single-mode spontaneous emission in a photonic nanowire," Phys. Rev. Lett. 108, 077405 (2012)

32. P. Lalanne, J. P. Hugonin, and J. M. Gérard, "Electromagnetic study of the quality factor of pillar microcavities in the small diameter limit," Appl. Phys. Lett. 84, 4726-4728 (2004). 